BULLETIN OF THE

AMERICAN MATHEMATICAL SOCIETY

Volume 77, Number 4, July 1971

\title{
ON THE SELBERG CONDITION FOR SUBGROUPS OF SOLVABLE LIE GROUPS
}

\author{
BY RICHARD TOLIMIERI
}

Communicated by L. Auslander, February 4, 1971

1. Introduction. In [1], S. P. Wang uses the techniques of [2] to prove a converse to a Selberg lemma for solvable groups. In [3], the author gave an elementary proof of the main result of [2] using the semisimple splitting. It is quite natural to expect that the results of [1] should also have an elementary proof in terms of the semisimple splitting. We do so in this paper.

2. Preliminaries. Let $S$ be a simply connected solvable analytic group with nil-radical $H$. Suppose we imbed $S$ as a subgroup of $\mathrm{GL}(n, R)$, the group of all $n$ by $n$ nonsingular real matrices. Let $\mathbb{Q}(S)$ denote the algebraic hull of $S$. Then we can write $Q(S)=N \cdot T$, where $N$ is the group of all unipotent matrices in $Q(S)$ and $T$ is a maximal abelian subgroup of semisimple matrices in $Q(S)$. The relevant facts about algebraic groups can be found in [6]. In [3] we stated the following result primarily due to L. Auslander [4].

There exists an imbedding of $S$ as a subgroup of $\operatorname{GL}(n, R)$ satisfying the following properties.

1. $H \subset N$.

2. The projection mapping $P: Q(S) \rightarrow N$ restricted to $S$ defines a diffeomorphism of $S$ onto $N$. We denoted the restriction of $P$ to $S$ by $n: S \rightarrow N$.

Denote the projection mapping of $a(S)$ into $T$ by $t$. Let $C$ be a closed subgroup of $S$. As we have seen in [3] we can choose $T$ so that $t(C) \subset a(C)$. Let $C^{\prime}=\mathfrak{Q}(C \cap H) C$. Then $C^{\prime} / C$ is compact and $C^{\prime}$ is closed in $S$. From our choice of $T$ and since $[a(C), a(C)] \subset a(C \cap H)$ it follows that $n\left(C^{\prime}\right)$ is a closed subgroup of $N$. It is easy to see that the following statements are equivalent.

1. $S / C$ is compact.

2. $S / C^{\prime}$ is compact.

3. $N / n\left(C^{\prime}\right)$ is compact.

4. $N=\mathfrak{Q}\left(n\left(C^{\prime}\right)\right)$.

AMS 1970 subject classifications. Primary 22E25, 22E40, 20G20.

Key words and phrases. Solvable, nilpotent, Lie group, topological group, algebraic hull, matrix groups, unipotent, semisimple splitting.

Copyright (C) 1971, American Mathematical Society 
3. Wang's Theorem A. We shall assume the following two simple lemmas from [1].

LEMma 3.1. Let $V$ be a finite-dimensional vector space over the reals, $W$ a proper subspace of $V$ and $G$ a connected solvable subgroup of $\mathrm{GL}(V)$. Then there is a neighborhood $\Omega$ of the identity in $G$ such that $\mathrm{U}_{o \in \Omega} g(W)$ $\neq V$.

Lemma 3.2. Let $X$ be a nonempty conic open subset of $V$ and $\Omega$ a compact neighborhood of 0 in $V$. Then for every $x$ in $X$, there is a positive number $r$ such that $s x+\Omega \subset X$ for all $s \geqq r$.

Let $C$ be a closed subgroup of the simply connected solvable analytic group $S$. We shall assume the notation and conventions of $\$ 2$.

We say that $C$ has the Selberg property in $S$ if and only if for any $s$ in $S$ and for any neighborhood $\Omega$ of 1 in $S$ there exists $u$ and $v$ in $\Omega$ and an integer $l>0$ such that $u s^{l} v$ is in $C$.

Theorem A. Suppose that $C$ has the Selberg property in $S$. Then $S / C$ is compact.

Proof. By 1.2 of [5], $C^{\prime}$ has the Selberg property. Since $S / C$ is compact if and only if $S / C^{\prime}$ is compact we can replace $C$ by $C^{\prime}$ in our discussion. Thus without loss of generality we will assume throughout that the subgroup $C$ under consideration has the addition property that $Q(C \cap H) \subset C$. It follows that $n(C)$ is a subgroup of $N$. We must prove that $N=Q(n(C))$.

Assume that $N$ is abelian. Suppose that $N \neq Q(n(C))$. Let $\Omega_{1}$ be the set of all $x$ in $N$ such the euclidean absolute value of $x$ is less than one. Let $\Omega_{2}$ be the set of all $x$ in $N$ whose euclidean absolute value is less than one half. Since $S$ normalizes $N$, by Lemma 1 it is easy to see that there is a compact symmetric neighborhood $\Omega$ of 1 in $S$ such that

(a) $\bigcup_{u \in \Omega} u Q(n(C)) u^{-1} \neq N$.

It follows from elementary topological group theory techniques (see p. 95 of [7]) that $\Omega$ can be chosen with the addition properties that

(b) For all $u$ in $\Omega, u \Omega_{2} u^{-1} \subset \Omega_{1}$.

(c) $\Omega^{2} C n^{-1}\left(\Omega_{2}\right)$ where $n$ is the homeomorphism of $S$ onto $N$ introduced in $\$ 2$.

By [5], $H C / H$ has the Selberg property in the vector group $S / H$. Thus by [5] again we have that $S / H C^{-}$is compact. It follows from our previous discussion that $N=H+Q(n(C))$. 
Let $X=N-\bigcup_{u \in \Omega} u Q(n(C)) u^{-1}$. Then $X$ is a nonempty open conic. It follows that there is an $h$ in $H$ such that $h^{l}+\Omega_{1} \subset X$, for all positive integers $l$. Since $C$ has the Selberg property in $S$ there are elements $u$ and $v$ in $\Omega$ and a positive integer $l$ such that $u h^{l} v$ is in $C$. Thus $u h^{2} u^{-1} n(u v)$ is in $n(C)$. Note that by $n(u v)$ we mean the application of the projection map $n$ to the product $u v$. From this equation we get that $h^{l} u^{-1} n(u v) u$ is in $u^{-1} n(C) u \nsubseteq X$. Our choice of $\Omega$ gives $u^{-1} n(u v) u$ $\in \Omega_{1}$. Thus $h^{l}+\Omega_{1} \nsubseteq X$, a contradiction.

Suppose $N$ is not abelian. Let $[x, y]$ denote the commutator $x y x^{-1} y^{-1}$ of $x$ and $y$. Denote the last nontrivial term in the lower central series of $N$ by $M$. Using induction on the number of terms of the lower central series of $N$ we can assume that the theorem holds in the group $S / M$. By [5], $M C / M$ has the Selberg property in $S / M$. Thus $N=M+Q(n(C))$.

Let $z$ and $z^{\prime}$ be in $N$. We can write $z=x y$ and $z^{\prime}=x^{\prime} y^{\prime}$ where $x$ and $x^{\prime}$ are in $M$ and $y$ and $y^{\prime}$ are in $\mathscr{Q}(n(C))$. Since $M$ is central in $N,\left[z, z^{\prime}\right]$ $=\left[y, y^{\prime}\right]$ is in $Q(n(C))$. Thus $M \subset[N, N] \subset \propto(n(C))$.

4. Wang's Theorem B. We shall be satisfied with proving Wang's Theorem B for the special case of $S$ simply connected.

TheOREm B. Let $S$ be a simply connected solvable analytic group, $C$ a discrete subgroup of $S$ such that $S / C$ is compact, and $Z$ the centralizer of $C$ in $S$. Then $Z$ is abelian.

Proof. Since $Z$ commutes with $C$ it commutes with $\mathscr{Q}(C)$. Thus $Z$ acts by the identity map on $N$. This easily implies that $Z$ must be contained in $N$. Thus $Z$ is abelian.

\section{REFERENCES}

1. S. P. Wang, On S-subgroups of solvable Lie groups, Amer. J. Math. 92 (1970), 389-397.

2. G. D. Mostow, Factor spaces of solvable groups, Ann. of Math (2) 60 (1954), 127. MR 15, 853.

3. R. Tolimieri, Applications of the semisimple splitting, Bull. Amer. Math. Soc. 77 (1971), 275-280.

4. L. Auslander and J. Brezin, Almost algebraic Lie algebras, J. Algebra 8 (1968), 295-313. MR 37 \#334.

5. A. Borel, Density properties for certain subgroups of semi-simple groups without compact components, Ann. of Math. (2) 72 (1960), 179-188. MR 23 \#A964.

6. G. D. Mostow, Fully reducible subgroups of algebraic groups, Amer. J. Math. 78 (1956), 200-221. MR 19, 1181.

7. L. S. Pontrjagin, Topological groups, GITTL, Moscow, 1954; English transl., Gordon and Breach, New York, 1966. MR 17, 171; MR 34 \#1439.

Yale University, New Haven, Connecticut 06520 